Cuadro 1. Especies propagadas en invernadero, su estado de conservación y resultados parciales de propagación, por semillas y en forma vegetativa.*

Table 1. Greenhouse propagated species, their state of conservation and partial results of vegetative and seed propagation.

\begin{tabular}{|c|c|c|c|}
\hline & $\begin{array}{c}\text { Estado de } \\
\text { Conservación }\end{array}$ & $\begin{array}{c}\text { Germinación } \\
(\%)\end{array}$ & $\begin{array}{c}\text { Brotación } \\
(\%)\end{array}$ \\
\hline \multicolumn{4}{|l|}{ Alliaceae } \\
\hline Gethyum cuspidatum & Vulnerable & 3,0 & 21,7 \\
\hline $\begin{array}{l}\text { Leucocoryne purpurea } \\
\text { Iridaceae }\end{array}$ & Vulnerable & & 90,9 \\
\hline Tigridia philipiana & Rara & 12,0 & \\
\hline \multicolumn{4}{|l|}{ Amaryllidaceae } \\
\hline Placea amoena & Vulnerable & $98,0^{*}$ & 64,3 \\
\hline Placea arzae & Vulnerable & $98,7^{*}$ & 80,0 \\
\hline Placea davidii & Rara & $84,7^{\star}$ & 100 \\
\hline Placea aff. germanii & Rara & & 90,9 \\
\hline Placea grandiflora & No evaluada & $88,7^{*}$ & 100 \\
\hline Placea lutea & No evaluada & $81,9^{*}$ & 100 \\
\hline Placea ornata & Rara & $95,2^{*}$ & 100 \\
\hline $\begin{array}{l}\text { Placea sp. } \\
\text { Papilionaceae }\end{array}$ & No evaluada & 64,0 & 97,4 \\
\hline Adesmia resinosa & Rara & 29,4 & \\
\hline Adesmia balsamica & Rara & 4,3 & \\
\hline Dalea azurea & En Peligro & 91,7 & 91,6 \\
\hline
\end{tabular}

\section{BIBLIOGRAFÍA}

GOLD, K.; LEÓN-LOBOS, P.; WAY. M. 2004. Manual de recolección de semillas de plantas silvestres para conservación a largo plazo y restauración ecológica. Instituto de Investigaciones Agropecua- rias, Centro Regional de Investigación Intihuasi, La Serena, Chile. Boletín INIA No $110,62 \mathrm{p}$. MYERS, N.; MITTERMEIER, R.A.; MITTERMEIER, C.G.; FONSECA, G.; KENT. J. 2000. Biodiversity Hotspot for conservation priorities. Nature 403: 853-858.

\title{
EVALUACIÓN DE PLANTAS NATIVAS PARA DECORAR NUESTROS JARDÍNES
}

\section{EVALUATION OF NATIVE PLANTS FOR EMBELLISHING OUR GARDENS}

Barrionuevo, V., Planchuelo, A.M y Fuentes, E.

Facultad de Ciencias Agropecuarias. Universidad Nacional de Córdoba, Argentina. Avda. Valparaíso s./n C.C. 509 - 5000 Córdoba-Argentina.

E-mail: vbbarrionuevo@yahoo.com.ar

\section{INTRODUCCIÓN}

La cobertura vegetal de la provincia de Córdoba es muy heterogénea, presentando unidades de vegetación que se encuentran muy degradadas (Cabido y Zac, 1999). Numerosas áreas de las sierras chicas han experimentado una gran pérdida de vegetación natural y de germoplasma 
nativo, como consecuencia del crecimiento urbano y de la extracción de plantas sin criterios conservacionistas. El valle de Paravachasca es un área turística que presenta una riqueza florística con especies de valioso potencial como ornamentales. El objetivo de este estudio es promover el conocimiento y la utilización sustentable de los recursos vegetales regionales, proponiendo la incorporación de especies de valor ornamental en espacios verdes y jardines regionales y urbanos.

\section{MATERIALES Y MÉTODOS}

Se realizaron viajes para evaluar la diversidad florística en distintos puntos del valle de $\mathrm{Pa}$ ravachasca y sus zonas aledañas (Los Reartes y Villa Berna), en distintas épocas del año. Se registraron características morfo-fisionómicas y de hábitat de las especies nativas que presentan valor ornamental y pueden ser de interés para su introducción en parques y jardines. Todas las especies se encuentran documentadas mediante ejemplares de herbario y están catalogadas en el Herbario ACOR de la Facultad de Ciencias Agropecuarias. U.N. Córdoba. Las identificaciones se realizaron con bibliografía especializada y los nombres científicos fueron actualizados según Zuloaga et al. (1999). La colección se encuentra disponible como un recurso didáctico y para su intercambio o préstamo a otras instituciones afines que los solicitasen. Además se cuenta con colecciones de plantas vivas conservadas "in situ" y "ex situ" para ser utilizadas como proveedoras de material vegetal para su reproducción agámica o por simientes.

Se está llevando a cabo planes de multiplicación vegetativa utilizando diversos materiales

\section{Cuadro 1. Angiospermas}

Table 1. Angiosperms

\begin{tabular}{|c|c|}
\hline FAMILIA & ESPECIE \\
\hline Amarantaceae & $\begin{array}{l}\text { Gomphrena pulchella Mart. } \\
\text { Pfaffia gnaphaloides (L.f.) Mart. }\end{array}$ \\
\hline Asclepiadaceae & Amblyopetalum coccineum (Griseb.) Malme \\
\hline Asteraceae & $\begin{array}{l}\text { Gallardia megapotámica var. radiata (Griseb) Baker } \\
\text { Stevia satureiifolia (Lam.) Sch. Bip } \\
\text { Trichocline plicata D. Don ex Hooker et. Arnott }\end{array}$ \\
\hline Boraginaceae & $\begin{array}{l}\text { Echium plantagineum } \mathrm{L} \text {. } \\
\text { Heliotropium nicotianae flolium Poir }\end{array}$ \\
\hline Campanulaceae & Wahlembergia linarioides (Lam.) A.DC. \\
\hline Convolvulaceae & Evolvulus sericeus Sw. \\
\hline Iridiaceae & $\begin{array}{l}\text { Sisyrinchium megapotámicum Malme } \\
\text { Sisyrrinchium platense I.M.Johnst. } \\
\text { Sisyrrinchium chilense Hook. }\end{array}$ \\
\hline Leguminoseae & $\begin{array}{l}\text { Cologania ovaligolia Kunth. } \\
\text { Desmoduim uncinatum (Yacq.) DC }\end{array}$ \\
\hline Lythraceae & Cuphea glutinosa Cham \& Schltdl \\
\hline Malvaceae & $\begin{array}{l}\text { Gaya parviflora (Phil.) Krapov } \\
\text { Pavonia aurigloba Krapov. \& Cristóbal }\end{array}$ \\
\hline Oenotheraceae & Oenothera rosaea Aiton \\
\hline Poaceae & Eustachys disticophylla (Lag.) Nees \\
\hline Polygalaceae & Monnina dictyocarpa Griseb. \\
\hline Portulaceae & $\begin{array}{l}\text { Portulaca grandiflora var. cresplatina Hook D. Legrand. } \\
\text { Portulaca perennis R.E. Fr. }\end{array}$ \\
\hline Solanaceae & $\begin{array}{l}\text { Nierembergia aristata D. Don } \\
\text { Petunia axillaris (Lam.) Britton, Stern \& Poggenb. }\end{array}$ \\
\hline Turneraeae & Turnera sidoide subsp. pinnatifide (Juss. ex Poir) Arbo \\
\hline Verbenaceae & $\begin{array}{l}\text { Glandularia peruviana (L.) Small } \\
\text { Glandularia platensis (Spreng.) Schnack \& Covas }\end{array}$ \\
\hline
\end{tabular}


de propagación (esquejes, división de matas, estolones) en especies de Pteridófitas y Fanerógamas, evaluando la eficiencia de las técnicas empleadas para incorporarlas a micro-emprendimientos regionales viveristas.

\section{RESULTADOS}

Hasta el momento se han catalogado más de cincuenta especies nativas con potencial ornamental, pertenecientes a 44 géneros y 22 familias de los grupos botánicos de las Pteridófiotas y Angiospermas. En el Cuadro 1 se presentan algunas de ellas.

Con la información recabada de cada especie se confeccionó un banco de datos sobre usos y propiedades de la flora regional autóctona y se diseñaron fichas técnicas que incluyen e ilustran los aspectos relevantes de cada una de ellas. Los datos suministrados en cada ficha son: nombres vernáculos, nombre científico, familia botánica, características morfo-fisonómicas atractivas, fotografías y esquemas ilustrativos, condiciones del hábitat y requerimientos de cultivo. Publicaciones preliminares (Barrionuevo, et al. 2004) muestran que la información de las fichas técnicas son de gran utilidad para caracterizar las especies ornamentales nativas y promover su utilización.

\section{CONCLUSIONES}

La caracterización y el conocimiento integral morfo-anatómico y los métodos de multiplicación de plantas nativas con potencial ornamental, constituyen el punto de partida para su reconocimiento y valoración.

La adaptación a los sistemas de cultivo favorece la propagación y permite encarar planes de mejoramiento vegetal. La difusión de sus cualidades y la venta de las plantas evitarán la extracción de su hábitat natural y brindará una importante fuente de trabajo a viveristas y aficionados al cultivo de plantas de las localidades serranas de Córdoba.

\section{BIBLIOGRAFÍA}

BARRIONUEVO, V. 2004. Evaluación de dos especies nativas de Glandularia para su cultivo como ornamentales. II Congreso Argentino de Floricultura y Plantas Ornamentales. I.N.T.A. Buenos Aires.

CABIDO, M.; ZAK. M. 1999. Vegetación del Norte de Córdoba. IMBIV, UNC- CONICET.

ZULOAGA, F. O.; MORRONE, O 1999. Catálogo de las Plantas Vasculares de la República Argentina. III Monogr. Syst. Bot. Missouri Bot. Gard. 60: $1-323$

Agro Sur 34 (1-2):47-48 2006

\title{
GERMINACIÓN DE CINCO ESPECIES NATIVAS CON VALOR ORNAMENTAL DE LA XII REGIÓN*
}

\section{GERMINATION OF FIVE NATIVE SPECIES FROM THE XII REGION, CHILE, WITH ORNAMENTAL VALUE}

\author{
Caicheo, A.', Vera, M.', Dollenz, O.', Yagello, J.' y Massardo, F. ${ }^{3}$ \\ 'Escuela de Recursos Naturales Renovables y Acuáticos, ${ }^{2}$ Departamento de Ciencias, ${ }^{3}$ Parque \\ Etnobotánico Omora, Sede Puerto Williams, Universidad de Magallanes; Correo Puerto \\ Williams, XII Región. E-mail: francisca.massardo@umag.cl
}

\section{INTRODUCCIÓN Y OBJETIVOS}

La Región de Magallanes y Antártica Chilena alberga numerosas especies arbustivas con enorme potencial ornamental. Entre éstas, dos Berberidaceae, el calafate (Berberís buxifolia) y el michay (B. ilicifolia) son arbustos considerados ornamentales gracias a su follaje brillan- te, llamativas flores amarillas polinizadas por insectos y bayas comestibles azules a negras consumidas por aves silvestres. Una Fabaceae arbustiva, el neneo o lengua de fuego (Anarthrophyllum desideratum) exhibe una peculiar forma de crecimiento de tipo cojín propia de la zona altoandina. Su atractiva arquitectura resalta aún más con el fuerte colorido y sincronía de 